\title{
BIOCHEMICAL AND HISTOLOGICAL EFFECTS OF TETRACYCLINES ON SPONTANEOUS OSTEOARTHRITIS IN GUINEA PIGS
}

\author{
EDIN DE BRI AND WEI LEI \\ Department of Orthopaedics, South Hospital, Karolinska Institute, 11883 Stockholm, Sweden \\ E-mail: de.bri@alfa.telenordia.se \\ (Accepted May 23, 2000)
}

\begin{abstract}
Matrix metalloproteinases (MMPs) are mediators in connective tissue destruction in a variety of pathologic processes. Recently discovered chemically modified tetracyclines have been found to be effective inhibitors of MMP mediated connective tissue degradation in both rheumatoid arthritis (RA) and osteoarthritis (OA). The Hartley guinea pig model has been described with a high incidence of spontaneous OA-like changes in the knee joint. Therefore we have studied the effect of two tetracyclines, doxycycline (Dox) and chemically modified tetracycline-7 (CMT-7) which have both previously been shown as potent MMP inhibitors. We found that prophylactic orally given CMT-7 decreases OA changes in the knee joints both in vitro and in vivo in the guinea pig OA model. OA changes were most severe in the central compartment of the medial condyle in the control group. Cartilage fibrillation and destruction, in addition to subchondral bone sclerosis and cyst formation were all less in the CMT-7 treated group compared with controls. Collagen, hyaluronan and proteoglycan content in cartilage was higher in the CMT-7 treated group compared with controls. In contrast, OA changes were not decreased in the Dox group. These results show that tetracyclines, but not all tetracyclines, can reduce the severity of OA in the guinea pig model of spontaneous OA.
\end{abstract}

Keywords: biochemistry, cartilage, guinea pig, osteoarthritis, stereology, tetracycline.

\section{INTRODUCTION}

A variety of matrix metalloproteinases (MMPs) especially collagenase and gelatinase, have been implicated in the connective tissue/cartilage degradation which characterizes osteoarthritis (OA) (Pelletier and Pelletier, 1996), also in animal models (Greenwald et al., 1990). A large body of literature has established that certain tetracyclines (TCs) are potent in vitro and/or in vivo inhibitors of various MMPs (Ryan et al., 1996). Doxycycline (Dox) is especially useful in this regard, and this agent has been shown to potently inhibit cartilage gelatinase (Cole et al., 1995), to have dramatic effects in the dog anterior cruciate OA model (Yu et al., 1993), and to reduce the excretion of collagen breakdown products in patients with rheumatoid arthritis (Greenwald et al., 1994). Chemically modified derivates which have been modified so as to eliminate the antimicrobial properties of the TC but preserve (and usually enhance) its MMP inhibitory capacity. The CMTs have been described previously (Ryan et al., 1996).
Light microscopic studies have shown that Hartley guinea pigs develop moderate to severe destruction of the cartilage and subchondral bone sclerosis between 6 and 12 months of age, predominantly in the central portion of the medial tibial plateau and that the changes regularly progress to severe OA in old age (de Bri et al., 1995; de Bri et al., 1996), with typical changes that mimic human disease (Bendele and Hulman, 1988).

Since the natural inter- and intra-animal variability is relatively large in normal cartilage, and even larger in pathological states, it is advantageous to study groups rather than individuals. This can be achieved by the use of stereology. In the present study, we have studied two TCs which have potent inhibitory capacity against various MMPs, doxycycline (Dox) and a compound known as chemically modified tetracycline-7 (CMT-7) (see below). These were given by mouth to a group of guinea pigs for 4 to 8 months and we subsequently assessed the effect of the compound on morphologic and biochemical aspects of $\mathrm{OA}$. 


\section{MATERIALS AND METHODS}

Hartley guinea pigs at age two months (approximately $350 \mathrm{gm}$ ) were used at the start of the experiment. A group was immediately sacrificed as baseline controls. One group was fed regular guinea pig chow ad lib and served as untreated controls. The remaining two groups were fed specially modified diets (Purina Test Diets, Richmond In, USA) containing the MMP inhibitors (see below) at a rate of $0.08 \% \mathrm{w} / \mathrm{w}$. Some animals in each group was sacrificed after 4 months (age 6 months) and the remainder at 8 months (age 10 months).

Two tetracycline (TC) compounds were used (CollaGenex Pharmaceuticals, Inc., Newtown PA, USA): doxycycline (Dox), and CMT-7 (12 $\alpha$ deoxy, 4-dedimethylaminotetracycline).

Since the protocol required daily administration of compounds for up to 8 months, it was elected to incorporate the drugs into the diet. Previous work had established that a daily dose of $20 \mathrm{mg} / \mathrm{kg}$ was an affective inhibitor of in vivo MMP activity (Greenwald et al., 1994).

\section{HISTOLOGY}

The specimens were fixed in a neutral-buffered $4 \%$ formalin, decalcified for $5-7$ days in $40 \%$ formic acid, cut into the medial and lateral plateaus, and embedded in paraffin wax. With random start, 4-6 histological sections were cut through each central portion of the plateau with a constant interval of $125 \mu \mathrm{m}$, and stained with hematoxylin and eosin. From these sections, volume densities $(\mathrm{Vv})$ of cartilage and bone were measured by point- and intersection counting $(>200$ hits per item and plateau) in a projection light microscope (ReichertJung, Germany) at a final magnification of $\mathrm{x} 50$. In this microscope, the image is projected onto a screen, on which the transparent grid (lattice) used for counting points and line intersections is attached. Cysts were defined as cavities larger than $100 \mu \mathrm{m}$ devoid of marrow cells, osteophytes as extra-articular osteocartilaginous tissue. Reference volume for cartilage, bone, and cysts was the entire epiphysis, i.e. the area bordered proximally by articular cartilage, anteriorly and posteriorly by cortical bone, and distally by the physeal remnant, osteophytes excluded. Cartilage fibrillation was measured by intersection counting with a cycloid grid for vertical sections (Baddeley et al., 1986) of the traced length of the contour of the cartilage surface and was divided by the length of the cartilage surface tangent.
The thickness of articular cartilage and subchondral bone plate was measured perpendicular to the joint surface; bone thickness was defined as the distance from the osteocartilaginous border to the first distal occurrence of nonosseous tissue. To control artifacts created by the procedure of tissue preparation, the type of fixative, time of fixation, $\mathrm{pH}$, osmolarity, dehydration, and embedding procedures were kept constant throughout the study. For statistical evaluation Students t-test at a rejection level of $\mathrm{p}<0.05$ was used. Stereological data are presented as ratios medial/lateral condyle.

\section{BIOCHEMISTRY}

Chondroitin sulfates (CS) (grade II preparation) and a high molecular weight hyaluronan (HA) standard were obtained from Sigma (St. Louis, MO, USA) and Pharmacia AB (Healon ${ }^{\circledR}$, Uppsala, Sweden) respectively. The aggrecan standard from chondrosarcoma was a generous gift from Prof. B. Caterson (Cardiff, U.K). The chondroitinase AC, chondroitinase $\mathrm{ABC}$, chondroitinase-6-sulfate, chondro- itinase-4-sulfate and disaccharide standards were obtained from Sigma (St. Louis, MO, USA). For chemical analysis of matrix components, 3 animals per group were used. The tibial articular cartilage was divided into two peripheral areas covered by the medial and lateral menisci and the two corresponding central uncovered areas, pooling material from the various animals. All analyses were performed in triplicate.

The tissue specimens were dissected, weighed and immediately frozen at $-20^{\circ} \mathrm{C}$, cut into $20 \mu \mathrm{m}$ thick slices, using a cryostat, lyophilized at $-50^{\circ} \mathrm{C}$ for $24 \mathrm{hrs}$, wherefore the dry weight was determined. The Proteoglycans (PG) were extracted with $4 \mathrm{M}$ guanidine hydrochloride (GuHCl), $\mathrm{pH} 5.8$ and containing $0.01 \mathrm{M}$ EDTA, $0.05 \mathrm{M}$ sodium acetate and proteinase inhibitors $0.1 \mathrm{M}$ 6-aminohexane, $5 \mathrm{mM}$ benzanhydrochloride, $5 \mathrm{mM}$ N-ethylmaleimide (the last mainly to prevent disulfide exchange). The extractions were performed at $4^{\circ} \mathrm{C}$ for $18 \mathrm{hrs}$, using $2 \times 40 \mathrm{ml}$ per mg dry tissue. The nonextracted residues were digested with papain and aliquots of these digests were hydrolyzed in $6 \mathrm{M} \mathrm{HCI}$ for $18 \mathrm{~h}$ and analyzed for their hydroxyproline contents. Large and small (PG) were separated electrophoretically. Ethanol precipitates of the extracts were dissolved in a SDScontaining electrophoresis buffer and run in $1.2 \%$ agarose gels at $90 \mathrm{~V}$ for $1.5 \mathrm{~h}$. The gels were stained with toluidine blue, scanning the distribution of PGs using a Shimadzu Dual-Wavelength Chromato- 
Scanner Model CS-930. The aggregability of the PG monomers was monitored by also incubating with HA before electrophoresis and comparing the mobility with that of preparations in which the HAbinding regions had been reduced. High-performance liquid chromatography (HPLC) was used to quantify the contents of CS and HA and to characterize the sulfation pattern. Aliquots were incubated with chondroitinase $\mathrm{AC}$ and chondroitinase $\mathrm{ABC}$, and the sulfation pattern was monitored by separating the delta-disaccharides obtained by HPLC, using external standards. The total amounts of CS and HA in these digests were determined following a further digestion with chondroitinase-4- and -6-sulfatases. The nonsulfated delta-disaccharides obtained from the respective GAGs were separated by ion suppression HPLC.

\section{RESULTS}

No signs of OA were discernable in the four animals that were immediately sacrificed at two months of age. In addition no OA changes were found macroscopically or on histological examination in the control, Dox and CMT-7 groups.

At 10 months, all animals in the control and Dox groups had developed advanced OA lesions in the central (meniscus non-covered) part of the medial condyle, while the peripheral (meniscus covered) part of the medial condyle was virtually non-affected by OA. In addition, the lateral condyle showed no macroscopic signs of OA. The lesions included cartilage destruction and occasional eburnation of the underlying calcified cartilage and subchondral bone. The CMT-7-treated guinea pigs showed only mild signs of OA, including surface fibrillation but without cartilage destruction and eburnation in the medial condyle, while the lateral condyle was unaffected by OA macroscopically.

\section{HISTOLOGY}

The guinea pigs in the control and Dox groups exhibited cartilage destruction, thereby exposing the calcified cartilage and occasionally the subchondral bone in the central (non-meniscus covered) compartment of the medial condyle. Also horizontal separation of the tidemark was observed in areas adjacent to cartilage destruction. The underlying trabecular structure of the subchondral bone plate was disturbed, and was replaced by an increased bone formation or bone sclerosis, and subsequently less bone marrow cavities, in addition to a thick subchondal bone plate. Cysts were frequently encountered in the sclerotic bone. Adjacent to the destructed cartilage, a transitional zone with fibrillation of the cartilage surface containing fewer chondrocytes, and separation of the uncalcified and calcified cartilage at the tidemark level was noticed. Only a few of the guinea pigs in the control group exhibited osteophytes at the joint margins, while the doxycycline- and CMT-7- treated animals showed no osteophytes. In the CMT-7-treated group, all guinea pigs had a milder form of OA, including fibrillation of the cartilage surface, but excluding overt destruction of the cartilage. Moreover, no cysts were encountered, and the trabecular structure of the subchondral bone was preserved and the subchondal bone plate was thinner. In addition, there were no signs of calcified cartilage eburnation or horizontal separation of the tidemark. The peripheral compartment of the medial condyle showed no signs of OA. No evidence of $\mathrm{OA}$ was observed in the central (meniscus non-covered) condyles in neither group.

\section{STEREOLOGY}

Stereological data are given as ratios medial/ lateral condyle since OA changes occurred only in the medial condyle, the lateral condyle thus serving as an internal control. The CMT-7-treated guinea pigs had lower $\mathrm{Vv}$ of bone, but higher $\mathrm{Vv}$ cartilage compared to the Dox group, however not reaching statistical significance compared to the control group (Table 1). There were no differences between the Dox group and the control group. Cartilage fibrillation was lower in the CMT-7-treated guinea pigs compared to both the Dox and control groups. In contrast there were no differences between the Dox group and the control group. Also the thickness of the cartilage was higher, and the thickness of the subchondral bone plate was lower in the CMT-7treated guinea pigs compared to both Dox and control groups (Table 1). 
Table 1. Guinea pigs treated with doxycycline (Dox) and chemically modified tetracycline-7 (CMT-7) are compared with untreated controls.

\begin{tabular}{llll}
\hline Group & Control $(\mathrm{n}=6)$ & Dox $(\mathrm{n}=6)$ & CMT-7 $(\mathrm{n}=4)$ \\
\hline M/L Vvbone & $1.46(0.18)$ & $1.38(0.11)$ & $1.12(0.12)^{\mathrm{a}, \mathrm{b}}$ \\
M/L Vvcartilage & $0.89(0.19)$ & $0.90(0.09)$ & $1.05(0.04)^{\mathrm{b}}$ \\
M/L Thcart & $0.91(0.07)$ & $0.90(0.07)$ & $1.00(0.12)$ \\
M/L Thbone & $1.90(0.13)$ & $2.00(0.34)$ & $1.39(0.16)^{\mathrm{a}, \mathrm{b}}$ \\
M/L fibrillation & $1.57(0.36)$ & $1.60(0.25)$ & $1.08(0.04)^{\mathrm{a}, \mathrm{b}}$ \\
\hline
\end{tabular}

$\mathrm{a}=\mathrm{p}<0.05$ between CMT-7 group and control group, $\mathrm{b}=\mathrm{p}<0.05$ between CMT-7 group and doxycycline group. Volume densities of bone (Vvbone) and cartilage (Vvcartilage), the thickness of articular cartilage (Thcart) and subchondral bone (Thbone) in addition to cartilage fibrillation (fibrill) were measured in the central medial and lateral condyles separately. The ratios medial/lateral condyle are given as means (SD).

\section{BIOCHEMISTRY}

Chondroitin sulfate (CS) was the major glycosaminoglycan (GAG) found in the articular cartilage. Total proteoglycan (PG) content, expressed as CS-derived uronic acid, was higher in the medial central condyle in the CMT-7-treated group compared to the control group. No differences were observed in the Dox group (Table 2). The amounts of both large and small PGs were also relatively higher in the central medial condyle in the CMT-7 group (Table 2). The highest PG concentration levels were found centrally in the medial condyle, while lower levels were found in the lateral condyles. The two articular cartilage fractions representing tissue covered by the menisci (peripheral compartment) contained considerably lower levels of PGs than their central counterparts.

Overall, the CS of unmineralized cartilage showed a predominance of 6-sulfated disaccharides (Table 3), with a considerable proportion of 4sulfated disaccharides (Table 3), leaving a minimal amount of non-sulfated disaccharides per chain. No oversulfated disaccharides were found. However, the sulfation pattern varied in the various areas of the tibial articular cartilage. We observed increased amounts of both $6 \mathrm{~S}$ and $4 \mathrm{~S}$, predominantly in the central medial condyle in the CMT-7 group compared with the Dox and control groups. However, the ratio of $6 \mathrm{~S} / 4 \mathrm{~S}$ was constant in the different groups and areas (Table 3).

Table 2. Guinea pigs treated with doxycycline (Dox) and chemically modified tetracycline-7 (CMT-7) are compared with untreated controls.

\begin{tabular}{llllll}
\hline Group & & & Control $(\mathrm{n}=6)$ & Dox $(\mathrm{n}=6)$ & CMT-7 $(\mathrm{n}=4)$ \\
\hline Chondroitin sulphate & Medial & Central & 17.7 & 14.2 & 33.6 \\
& & Peripheral & 21.4 & 17.3 & 32.2 \\
& Lateral & Central & 17.0 & 18.6 & 25.5 \\
& & Peripheral & 16.4 & 11.9 & 22.2 \\
\hline Large proteoglycans & \multirow{2}{*}{ Medial } & Central & 14.9 & 12.1 & 27.5 \\
& & Peripheral & 17.6 & 14.1 & 25.7 \\
& \multirow{2}{*}{ Lateral } & Central & 13.9 & 14.9 & 20.6 \\
& & Peripheral & 13.4 & 9.6 & 17.8 \\
\hline Small proteoglycans & \multirow{2}{*}{ Medial } & Central & 2.8 & 2.1 & 6.1 \\
& & Peripheral & 3.8 & 3.2 & 6.4 \\
& \multirow{2}{*}{ Lateral } & Central & 3.1 & 3.7 & 4.9 \\
& & Peripheral & 2.9 & 2.4 & 4.4 \\
\hline
\end{tabular}

The amounts of chondroitin sulphate, large proteoglycans are given for the central and peripheral compartments of the medial and lateral condyles, respectively ( $\mu \mathrm{g}$ CS-derived uronic acid per mg dry weight cartilage). 
Table 3. Guinea pigs treated with doxycycline (Dox) and chemically modified tetracycline-7 (CMT-7) are compared with untreated controls.

\begin{tabular}{|c|c|c|c|c|c|}
\hline Group & & & Control $(n=6)$ & Dox $(n=6)$ & CMT-7 $(n=4)$ \\
\hline \multirow[t]{4}{*}{6 Sulphate } & Medial & Central & 10.1 & 8.5 & 21.0 \\
\hline & & Peripheral & 12.4 & 8.6 & 20.0 \\
\hline & Lateral & Central & 8.9 & 10.4 & 16.8 \\
\hline & & Peripheral & 10.0 & 6.6 & 10.0 \\
\hline \multirow[t]{4}{*}{ 4 Sulphate } & Medial & Central & 4.1 & 3.7 & 9.4 \\
\hline & & Peripheral & 5.2 & 3.7 & 10.1 \\
\hline & Lateral & Central & 4.1 & 4.5 & 7.3 \\
\hline & & Peripheral & 4.8 & 3.3 & 5.0 \\
\hline \multirow[t]{4}{*}{ 6/4 Sulphate } & Medial & Central & 2.4 & 2.3 & 2.2 \\
\hline & & Peripheral & 2.4 & 2.3 & 2.0 \\
\hline & Lateral & Central & 2.2 & 2.3 & 2.3 \\
\hline & & Peripheral & 2.1 & 2.0 & 2.0 \\
\hline
\end{tabular}

The amounts of proteoglycans sulphated in 6 and 4 position in addition to the $6 / 4$ sulphate ratio are given for the central and peripheral compartments of the medial and lateral condyles, respectively ( $\mu \mathrm{g}$ CS-derived uronic acid per mg dry weight cartilage).

The CS was extracted from the uncalcified tissue in the form of PGs which, by electrophoresis, could be separated into one smaller, faster-moving band and two dominating closely migrating but distinct bands with lower mobility, representing a larger molecular size. The faster moving band migrated slightly slower than the free CS chains, while the mobility of the large PG was similar to that of chondrosarcoma aggrecan. The mobility of the PG populations remaining in different fractions was similar in all three groups. Furthermore, the ratios of large to small PGs in the uncalcified tissue fractions were very similar in the three groups.
Collagen content, expressed as hydroxyproline, was higher in the medial central condyle in the CMT7-treated group compared to the control group, while no obvious difference was observed between the Dox group and the control group (Table 4).

The tissue contained a minor component of hyaluronan (HA). The concentration was higher in the central medial condyle in the CMT-7-treated group, compared to the control group (Table 4). No differences were observed between the Dox group and the control group. However, the ratios of large PG/HA were similar in all three groups. The aggregability was the same in all three groups.

Table 4. Guinea pigs treated with doxycycline (Dox) and chemically modified tetracycline-7 (CMT-7) are compared with untreated controls.

\begin{tabular}{|c|c|c|c|c|c|}
\hline Group & & & Control $(n=6)$ & Dox $(n=6)$ & CMT-7 $(\mathrm{n}=4)$ \\
\hline \multirow{4}{*}{ Hyaluronan } & Medial & Central & 0.5 & 0.6 & 1.0 \\
\hline & & Peripheral & 0.7 & 0.6 & 0.8 \\
\hline & Lateral & Central & 0.7 & 0.7 & 0.6 \\
\hline & & Peripheral & 0.6 & 0.4 & 0.6 \\
\hline \multirow[t]{4}{*}{ Hydroxyproline } & Medial & Central & 58 & 58 & 82 \\
\hline & & Peripheral & 53 & 56 & 58 \\
\hline & Lateral & Central & 56 & 62 & 62 \\
\hline & & Peripheral & 58 & 60 & 63 \\
\hline
\end{tabular}

The amounts of hyaluronan and hydroxyproline are given for the central and peripheral compartments of the medial and lateral condyles, respectively ( $\mu \mathrm{g}$ hyaluronan and hydroxyproline per mg dry weight cartilage).

\section{DISCUSSION}

Activated MMPs may play a role in the pathologic breakdown of the joint extracellular matrix in OA. TCs have been found to be effective inhibitors of MMP-mediated connective tissue destruction in a variety of pathologic processes, including rheumatoid arthritis and OA. The CMTs are potent inhibitors of several classes of matrix metalloproteinases, preventing collagen breakdown. We chose the Hartley guinea pigs, because of a previously reported high incidence of OA-like 
changes in the proximal tibia (Bendele and Hulman, 1988). The advantages of spontaneous OA models are evident. In many surgically induced models, it is difficult to control post-operative changes. It is known that the increased cytokine levels induced by trauma, and the subsequent joint inflammation, may have a direct effect on the development of OA, in contrast to spontaneous OA models, where the intensity of inflammation is low. Moreover, surgically induced OA models incompletely reproduce the slowly progressive course of primary OA. In secondary OA, the lesions develop rapidly, thereby differing from the slowly progressive disease process in primary $\mathrm{OA}$.

We observed that both histological and biochemical OA-changes in guinea pigs were less severe after 8 months of per oral treatment with CMT-7. The CMT-7 treated animals showed only mild fibrillation compared to the severe OA changes found in the control group including both cartilage destruction and subchondral bone sclerosis. These changes were most pronounced in the central medial condyle which is most commonly affected by OA (de Bri et al., 1995). Stereological parameters, allowing for quantitative data and comparisons between groups, showed lower $\mathrm{Vv}$ of both bone and cysts, in addition to a thinner subchondral bone plate, indicating less bone involvement. In contrast, Vv cartilage was higher, and the cartilage was thicker suggesting less destruction of the articular surface. Also fibrillation of the cartilage was lower in this group. Biochemically, total PG content (both small and large PGs), HA and collagen content in cartilage was higher in the CMT-7 treated animals compared to the OA affected control group suggesting a better preservation of articular cartilage. No differences were observed in the sulfation pattern with regards to the $6 \mathrm{~S} / 4 \mathrm{~S}$ ratio, although some studies have reported differently (Roughley and White, 1980). However the CMT-7 group had higher levels of both 6S and 4S. In $\mathrm{OA}$, the function of the joint cartilage is impaired due to matrix destruction, where proteoglycan content is decreased proportionally to the severity of OA (Venn and Maroudas, 1977). Interestingly there were no differences in OA changes between the Dox and the control group neither histologically nor biochemically in contrast to earlier findings. Brandt (1994) showed that in surgically induced OA in the dog model, prophylactic administered Dox ameliorated the induced pathologic changes. Active and latent collagenase and gelatinase levels in extracts of the OA knee joints were reduced due to Dox administration. However, in our hands, Dox was not effective, perhaps because the uptake after oral administration might have been impaired in contrast to CMT-7.

Naturally, these results have to be confirmed by a study with a larger sample size, and the uptake of the compounds has to be elucidated. Moreover, not only the prophylactic effect, but also the therapeutic effects of CMT-7 and Dox have to be evaluated.

In conclusion, prophylactic CMT-7 given orally decreases OA changes in the knee joints both in vitro and in vivo in the Guinea pig OA model. In contrast, Dox did not have any effect on the OA changes.

A preliminary report of some of the data was presented at the $X^{\text {th }}$ International Congress for Stereology, Melbourne, Australia, 1-4 November 1999.

\section{REFERENCES}

Baddeley AJ, Gundersen HJG, Cruz-Orive LM (1986). Estimation of surface area from vertical sections. J Microsc 142:259-76.

Bendele AM, Hulman JF (1988). Spontaneous cartilage degeneration in guinea pigs. Arthritis Rheum 31:561-5.

Brandt K (1994). Insights into the natural history of osteoarthritis provided by the cruciate-deficient dog. Ann NY Acad Sci 732:199-205.

Cole AA, Yi W, Kuettner K, Golub LM, Greenwald RA (1995). The effects of chemically modified tetracyclines on cartilage degradation in chicken tibial explants. Trans Orthop Res Soc 20:337.

De Bri E, Reinholt FP, Svensson O (1995). Primary osteoarthrosis in guinea pigs: A stereological study. J Orthop Res 13:769-76.

De Bri E, Jönsson K, Reinholt FP, Svensson O (1996). Focal destruction and remodelling in guinea pig arthrosis. Acta Orthop Scan 67(5):498-504.

Greenwald RA, Golub LM, Ramamurthy NS, McNamara $T$ (1990). Direct detection of collagenase and gelatinase in periarticular tissue from adjuvant arthritis rats: inhibition by tetracyclines and potential amelioration of bone destruction. Trans Orthop Res Soc 15:270.

Greenwald RA, Moak SA, Golub LM (1994). Low-dose doxycycline (LDD) inhibits pyridinoline (PYD) excretion in selected patients with rheumatoid arthritis. In Inhibition of Matrix Metalloproteinases: Therapeutic Potential, Ann NY Acad Sci 732:419-21.

Pelletier JM, Pelletier JP (1996). Wanted - the collagenase responsible for the destruction of the collagen network in human cartilage. Br J Rheum 35:818-20.

Roughley PJ, White RJ (1980). Age-related changes in the structure of proteoglycan subunits from human articular cartilage. J Biol Chem 255:217 24. 
Ryan ME, Ramamurthy NS, Golub LM (1996). Matrix metalloproteinases and their inhibition in periodontal treatment. Curr Opin Peridont 3:85-96.

Venn MF, Maroudas A (1997). Chemical composition and swelling of normal and osteoarthritic femoral head cartilage. I. Chemical composition. Ann Rheum Dis 36:121-9.

$\mathrm{Yu}$ LP, Smith GN, Brandt KD, Myers SL, O'Connor BL, Brandt DA (1992). Reduction of the severity of canine osteoarthritis by prophylactic treatment with oral doxycycline. Arthritis Rheum 35:1150-9. 